\title{
Kinetics in the gas adsorption process of porous coordination polymers by time-resolved $X$-ray powder diffraction measurement
}

\author{
H. Ashitani ${ }^{1}$, S. Kawaguchi ${ }^{2}$, H. Ishibashi ${ }^{1}$, K. Otake ${ }^{3}$, S. Kitagawa ${ }^{3}$ and Y. Kubota ${ }^{1}$ \\ ${ }^{1}$ Department of Physical Science, Osaka Prefecture University, Sakai, Osaka 599 8531, Japan \\ 2 Japan Synchrotron Radiation Research Institute (JASRI), SPring-8, Sayo, Hyogo, 679-5198, Japan \\ ${ }^{3}$ Institute for Integrated Cell-Material Sciences, Kyoto University, Kyoto 606-8501, Japan, \\ s_h.ashitani@p.s.osakafu-u.ac.jp
}

Porous coordination polymers (PCPs) with flexible framework have attracted much attention, because some of them have a high selective adsorption ability for specific gas molecules and a wide range of applications, e.g. gas separation, gas purification is expected. However, the mechanism of selective adsorption in PCPs is not unveiled. Crystal structure information of flexible framework and gas molecules is indispensable for clear understanding of the gas adsorption phenomena in PCPs.

In past decades, crystal structures of desorption and adsorption phases of PCPs were revealed by in-situ synchrotron powder diffraction measurements. Not only the framework but also the position and orientation of adsorbed gas molecules led to a deep understanding of the static gas adsorption state [1]. On the other hand, it is very interesting to know how the framework and/or pore surface of PCPs recognize gas molecules during the gas adsorption process. It will contribute to not only the development of PCPs with superior gas separation ability, but also the understanding of the whole gas adsorption process. However, there are few studies on the state at the beginning of gas adsorption process into PCPs. The dynamic structural information, which is the structural change of the gas molecules and framework in the overall gas adsorption process, will make it possible for us to gain the knowledge how gas molecules begin to interact with the pore surface and subsequently diffuse into the pores. In general, it is not so easy to elucidate the information on the early stage of the gas adsorption process by conventional measurement methods such as the adsorption isotherm. In order to obtain the dynamic structural information, we performed time-resolved synchrotron X-ray powder diffraction (XRPD) experiment in the gas adsorption process on PCPs.

In this study, XRPD measurements under gas pressure control were performed using the remote gas and vaper pressure control (RGVPC) system at beamline BL02B2 of SPring-8. The RGVPC system can control the gas and vaper pressure in online, and synchronize it with the powder diffraction data acquisition to obtain time-resolved data [2]. Using this system, a fixed amount of gas can immediately be introduced to a powder sample in a glass capillary (gas-shot mode). In the time-resolved XRPD measurement, the exposure time was set to be $1 \mathrm{~s}$ for each measurement. Previous to the measurement, powder samples are heated evacuating to remove guest molecules in the pore. After that, the temperature is lowered to $195 \mathrm{~K}$ and gas-shot measurement for $\mathrm{CO}_{2}$ gas started. Such measurements were performed by changing gas pressure and temperature. Figure 1 shows one of the changes of XRPD pattern of gas-shot measurement for $\mathrm{CO}_{2}$ gas adsorption in CPL-1 [3]. It was found that the $\mathrm{CO}_{2}$ adsorption completed within a few tens of seconds after the introduction of gas. In order to investigate the change of crystal lattice during this adsorption process, Le Bail fitting was performed for each timeresolved XRPD data. The speed changes of the lattice parameters were slightly different for each axis. Furthermore, the transformed fraction from desorption to adsorption phase was derived from the change of integrated intensity of specific diffraction peak. The results show that the transformed fraction strongly depends on the gas pressure and temperature. Its fraction might be related with the dimensionality of gas diffusion into the pores. These data were analyzed using kinetic method such as the Kolmogorov-Johnson-MehlAvrami (KJMS) theory [4,5]. In the presentation, we will discuss the kinetics and the structural change in gas adsorption process in comparison with PCPs with different pore size and shape.

[1] Kitaura, R., Kitagawa, S., Kubota, Y., Kobayashi T.C., Kindo, K., Mita, Y., Matsuo, A., Kobayashi, M., Chang, H., Ozawa, T., Suzuki, M., Sakata, M. \& Takata, M. (2002). Science 298, 2358-2361.

[2] Kawaguchi, S., Takemoto, M, Tanaka, H., Hiraide, S., Sugimoto, K., \& Kubota, Y. (2020). J. Synchrotron Rad. 27, 616-624.

[3] Kondo, M., Okubo, T., Asami, A., Noro, S., Yoshitomi, T., Kitagawa, S., Ishii, T., Matsuzaka, H., \& Seki, K. (1999). Angew. Chem. Int. Ed. 38, 140-143.

[4] Avrami, M. (1939). J. Chem. Phys. 7, 1103-1112.

[5] Kruger, P. (1993). J. Phys. Chem. Solids 54, 1549-1555. 


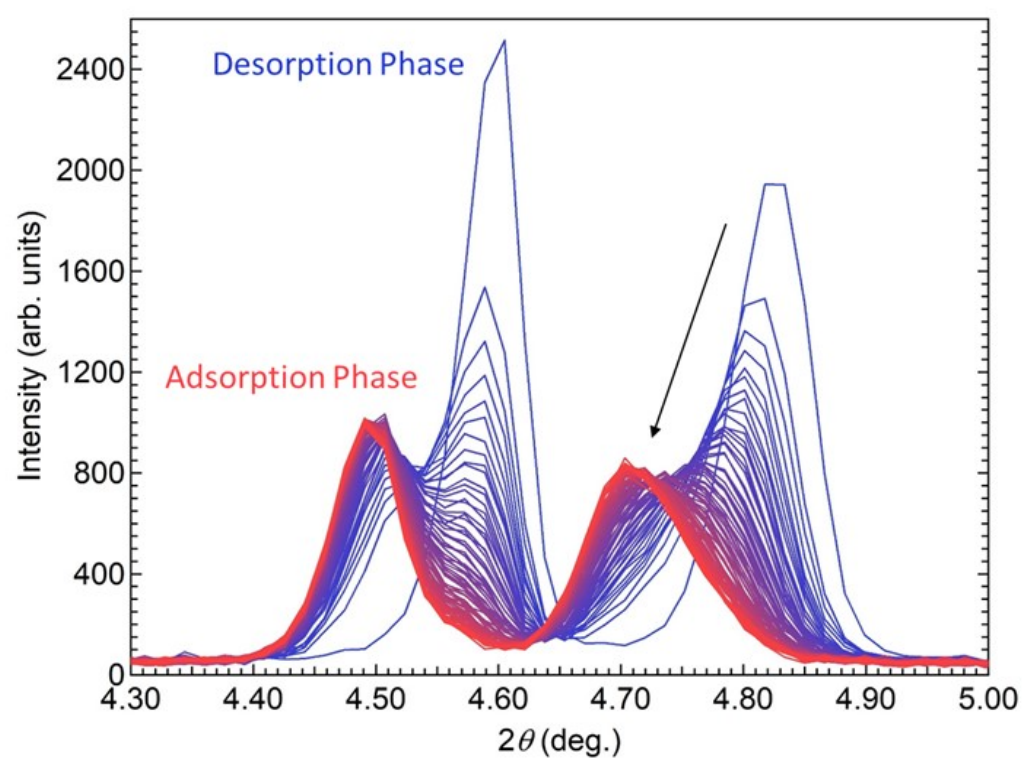

Figure 1. Change in XRPD profile measured by gas-shot mode in the CO2 adsorption in CPL-1 at 195K. The introduced gas pressure was $39 \mathrm{kPa}$. Data between gas introduction (Blue) and after $80 \mathrm{~s}$ (Red) are shown.

Keywords: powder diffraction; time-resolved measurement; porous coordination polymers; metal organic frameworks

This research was supported by KAKENHI Grant Nos. (20H04466, 20H02575). The synchrotron radiation experiments were performed at beamline BL02B2 of SPring-8 with the approval of the Japan Synchrotron Radiation Research Institute (JASRI) (Proposal Nos. 2020A0068, 2021A0068). The authors thank Mr. M. Takemoto for their assistance in the time-resolved powder diffraction measurement. 